Revue des patrimoines

16 | 2011

Le patrimoine militaire et la question urbaine

\title{
Patrimonialiser les bases de sous-marins et le Mur de l'Atlantique
}

\section{Claude Prelorenzo}

\section{(2) OpenEdition}

Journals

Édition électronique

URL : http://journals.openedition.org/insitu/312

DOI : 10.4000/insitu.312

ISSN : $1630-7305$

Éditeur

Ministère de la culture

Référence électronique

Claude Prelorenzo, «Patrimonialiser les bases de sous-marins et le Mur de l'Atlantique », In Situ [En ligne], 16 | 2011, mis en ligne le 01 septembre 2014, consulté le 30 avril 2019. URL : http:// journals.openedition.org/insitu/312; DOI : 10.4000/insitu.312

Ce document a été généré automatiquement le 30 avril 2019

\section{(c) $($ i) $(9)$}

In Situ Revues des patrimoines est mis à disposition selon les termes de la licence Creative Commons Attribution - Pas d'Utilisation Commerciale - Pas de Modification 4.0 International. 


\title{
Patrimonialiser les bases de sous- marins et le Mur de l'Atlantique
}

\author{
Claude Prelorenzo
}

1 Le Mur de l'Atlantique ${ }^{1}$ qui court de la frontière espagnole jusqu'au nord de la Norvège, est l'un des rares exemples d'un héritage bâti européen partagé puisqu'il concerne, outre la France où se trouve la majeure partie des fortifications réalisées, et l'Allemagne qui en fut le producteur, la Finlande, la Norvège, les Pays-Bas, la Belgique..., soit $1200 \mathrm{~km}$ de côtes fortifiées.

2 La construction du « Rempart Atlantique » est engagée dès 1940, avec les défenses du Cap Gris-Nez en France, avant même la signature formelle du cessez-le-feu ; elle se poursuivra jusqu'à la reddition du régime nazi, laissant inachevés des ouvrages importants comme, par exemple, la base de sous-marins de Marseille, pièce importante du « Mur Sud ». 


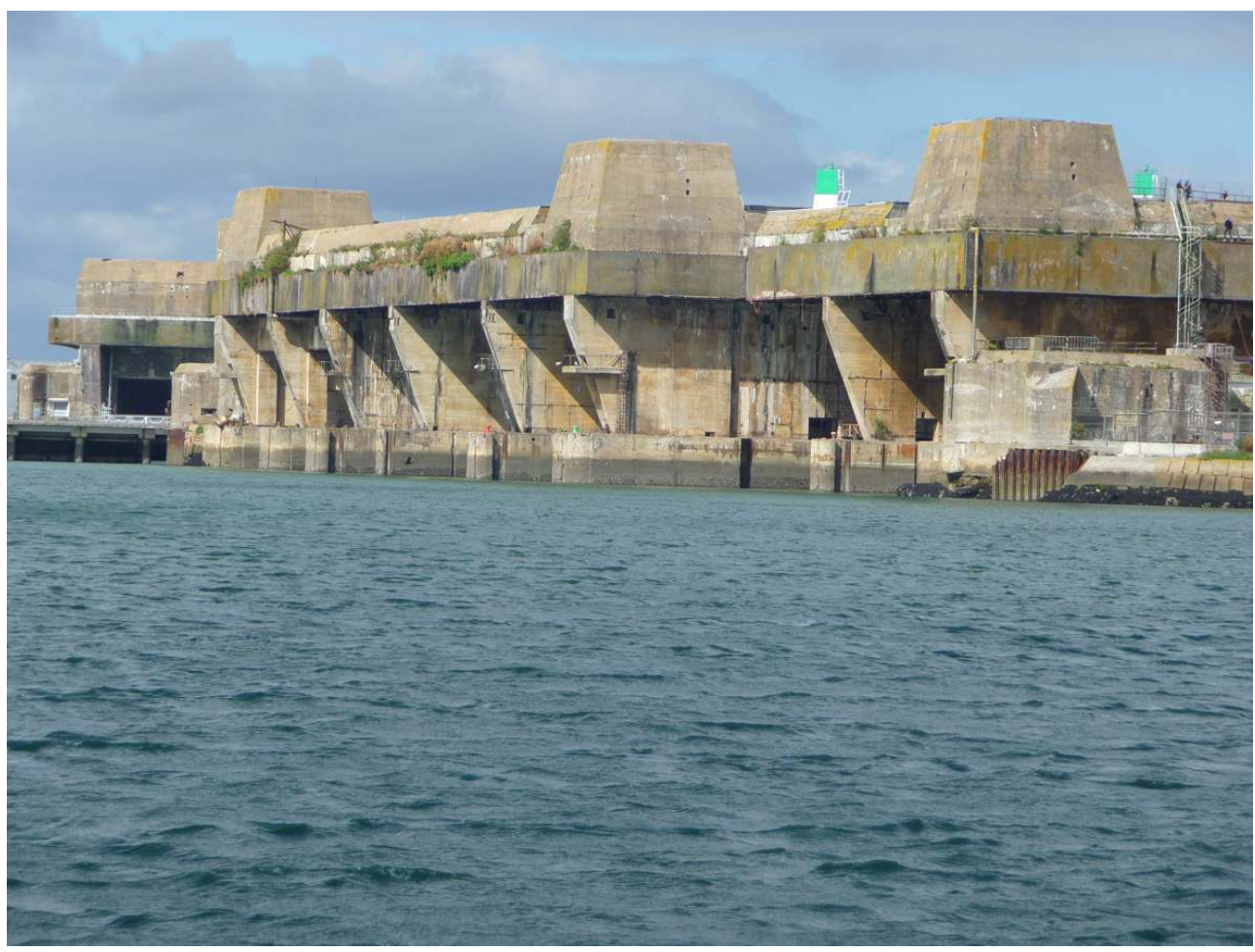

Base de sous-marins de Lorient. Façade du block à 7 alvéoles. Phot. Claude Prelorenzo.

3 Dans ce dispositif les bases de sous-marins ${ }^{2}$ occupent une place particulière (fig. $\mathbf{n}^{\circ} \mathbf{1}$ ). Au contraire de l'essentiel des constructions, les bases ne sont pas des équipements de défense et de surveillance, mais des instruments offensifs. Elles sont construites pour armer et entretenir une flottille de petits sous-marins, les U-Boats, lancés à l'assaut des Liberty Ship qui amènent en Angleterre hommes et matériels en vue du débarquement. Deuxième différence, au lieu d'être enterrées, camouflées et accompagnées de réseaux souterrains, les bases de sous-marins sont à l'extérieur et facilement identifiables par mer, terre ou ciel, leur protection étant assurée par de formidables toits à l'épreuve des bombes, par l'épaisseur du béton et par la couverture aérienne des batteries qui les entourent. Les sous-marins eux-mêmes sont protégés soit par une plongée rapide, comme à Lorient, soit par des écluses bétonnées, comme à Saint-Nazaire. Autre caractère qui n'est pas sans influence sur leur réutilisation pacifique, les bases sont situées dans ou contre les villes alors que le Mur en tant qu'entité marque des territoires souvent extraurbains, de dunes, de falaises, de plages, de champs, d'embouchures... Mais ces différences ne doivent pas faire oublier que les bases font partie intégrante du Mur et qu'elles ont de nombreux traits communs en tant que chantiers: elles furent construites par le même système Todt, elles sont du même béton que les autres fortifications, elles complètent également le dispositif défensif en en protégeant l'accès par la mer, etc.

Durant plusieurs décennies, les bases restent en-dehors des projets des édiles et de l'attention des citadins. En ruine et indestructibles, elles émettent un message difficile à admettre car c'est à cause d'elles que les villes furent détruites, parfois complètement comme à Lorient, et ces dommages furent causés non pas par l'ennemi mais par les Alliés dont les techniques de bombardement par nappe furent particulièrement destructrices, parfois sans grande justification stratégique, sinon de frapper de stupeur l'ennemi et 
obtenir une plus rapide reddition. C'est le mouvement des générations qui refoule la mémoire des témoins directs qui permet dans les années 1990 d'entreprendre la réinsertion de ces grandes structures de béton. Les jeunes générations n'y lisent plus seulement la destruction de la ville, mais y trouvent un matériau à des rêveries d'urbanités inusuelles: patinoires, fêtes, salles de sport qui deviendront en termes édilitaires musées, lieux d'expositions, de manifestations éphémères, promenades avec en particulier des belvédères sur les toits...(fig. $\mathbf{n}^{\circ} \mathbf{2}$ )

Figure 2

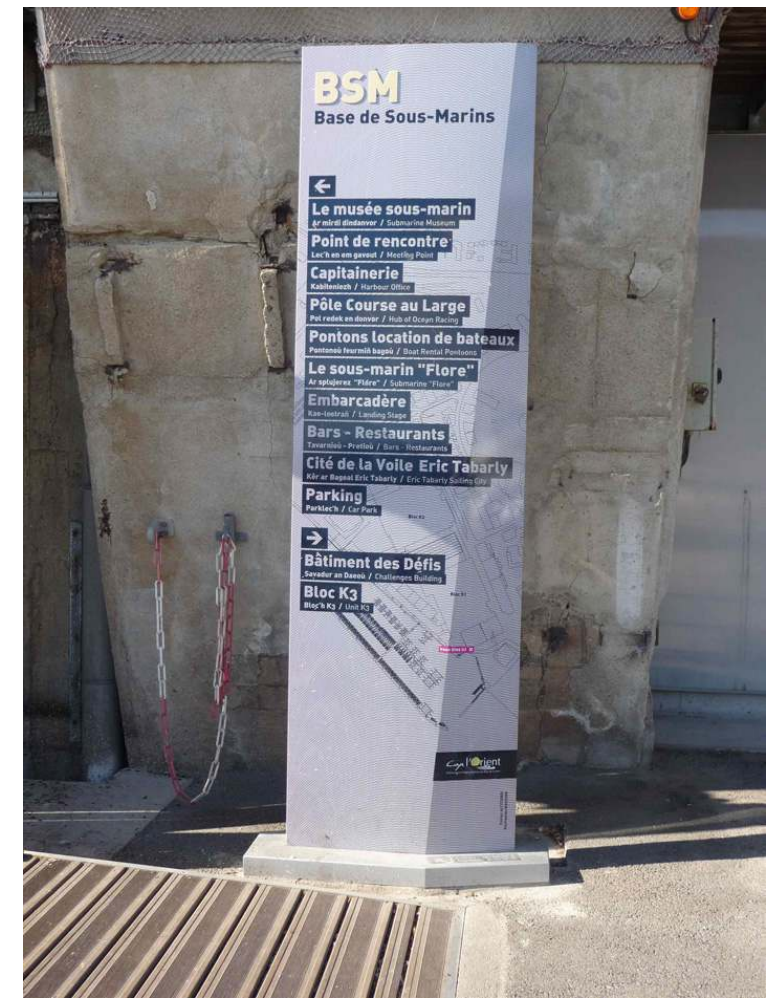

Base de sous-marins de Lorient. Borne d'information. Phot. Claude Prelorenzo.

5 C'est dans cette évolution que la patrimonialisation des constructions s'élabore, en deçà et au-delà de la trivialité des programmes culture + loisirs + commerce.

6 Nous chercherons donc dans cet article à identifier les valeurs culturelles qui justifient le maintien et la rénovation des bases. De même nous évoquerons le Mur en lui-même car c'est l'ensemble qui constitue la mémoire et sa patrimonialisation. C'est le sens de la proposition de création, au moins sous une forme immatérielle, d'un Musée linéaire du Mur de l'Atlantique ${ }^{3}$.

\section{Le Mur de l'Atlantique dans la modernité}

7 S'il relève avant tout du génie militaire, le Mur ne saurait être complément indépendant des systèmes constructifs, des matériaux disponibles et des techniques mises en œuvre, et même plus profondément des catégories de la conception architecturale et des paramètres de l'usage des espaces. On doit se souvenir ${ }^{4}$, à ce propos, que c'est Albert Speer, architecte du régime, et alors Ministre de la production de guerre et de la 
production, qui propose Fritz Todt à Hitler. À la mort de Todt en 1942, Speer se charge de poursuivre la construction des bases maritimes. Or Speer est très conscient dans ses propres réalisations comme dans ses avis et autorisations de l'efficience de l'esthétique architecturale tout autant que de la durabilité de l'œuvre qui doit porter témoignage pour les siècles futurs de la légitimité du III ${ }^{e}$ Reich. On retrouve cette attitude dans l'insistance "publicitaire» que signale Andrea Santangelo ${ }^{5}$ d'après lequel «son obsolescence fonctionnelle survint avec une rapidité unique dans l'histoire de la fortification moderne $»^{6}$. Cette volonté de populariser le Mur et de le valoriser indique que le " produit » avait des qualités qui permettaient de susciter une attention favorable dont on espérait qu'elle atténuerait l'hostilité que ne manqueraient pas de susciter l'étalage de la puissance militaire et son occupation violente et visible des territoires nationaux. Si la propagande autour du Mur s'appuie avant tout sur l'exploit technique (rapidité d'exécution) et sur ampleur territoriale inusitée du dispositif. Il fallait toutefois montrer quelques images dans les magazines et sur les écrans. Ce sont donc les «objets » euxmêmes qui seront mis en valeur, les cartes et les plans restant alors, comme aujourd'hui, des documents pour spécialistes. La pertinence architecturale du Mur est dès lors instituée et le Mur, malgré les connotations "romaines $»^{7}$ de ce type de "muraille», s'organise à partir du couple industriel/modernité des formes.

Quand bien même le Mur n'aurait pas été enveloppé dans ce commentaire propagandiste, on ne pourrait se dispenser d'interroger sa "modernité ", de le replacer dans l'histoire des formes architecturales car c'est aujourd'hui dans cette visée patrimoniale que se fonde un nouvel intérêt pour cet héritage guerrier. Les touristes qui visitent casemates, blockhaus et redoutes le long des côtes de l'Europe du Nord viennent pour être sur les lieux d'événements (peut-être de moins en moins "douloureux» mais toujours « importants »), pour se complaire dans le spectacle figé de la violence militaire, par goût romanesque de l'ancien qu'est déjà devenu le Mur, ne serait-ce que par ses ruines et ses patines, mais aussi pour sa modernité. Face à ce tourisme, difficile à canaliser, qui peut à tout moment dériver vers l'admiration de la puissance nazie, la mise en avant de la modernité architecturale n'est-elle qu'un refuge idéologique, un redoublement de la propagande nazie elle-même? En effet, mêlée aux sentiments plus ou moins troubles que nous avons évoqué (la visite des fortifications et des ouvrages qui composent le Mur), on constate une certaine forme d'acceptation de la morphologie moderne brutaliste. Les mêmes qui ne voient pas l'intérêt de visiter les Unités d'habitation de Le Corbusier ne restent pas insensibles à l'harmonie qui imprègne les formes douces, arrondies, féminines des blockhaus qui semblent s'abandonner sur le sable, sensuellement renversés parfois, ni au grain du béton usé, ou encore à leur fixité de balises attentives à la mer vide.

9 C'est pourquoi il nous a semblé utile de relever rapidement quelques éléments qui pourraient établir les paramètres de cette relation du Mur et de ses bases avec la modernité et le Mouvement moderne ${ }^{8}$.

\section{La meurtrière, la fenêtre en longueur et le cinémascope}

$10 \mathrm{Au}$ contraire de la fortification antique ou classique, le système de surveillance et d'angle de feu des blockhaus, des tours, des batteries, et des fortifications diverses privilégie la vision et le balayage horizontaux ${ }^{9}$.

11 Archères, meurtrières et même canonnières avaient instauré, depuis longtemps, le primat de l'ouverture verticale (parfois rondes pour certaines canonnières, très rarement 
horizontales). Dans le cas du Mur, si la fonction est la même (surveiller et faire feu en étant soi-même le moins visible possible et le plus protégé des tirs ennemis), le système est différent. Le plus souvent l'ouverture est horizontale. Et ceci la relie à deux phénomènes déterminants de la modernité de la seconde moitié $\mathrm{du} \mathrm{XX} \mathrm{X}^{\mathrm{e}}$ siècle : la fenêtre en longueur d'une part, la prééminence de l'écran horizontal au cinéma de l'autre, deux manières d'inventer, au-delà des cils, un nouveau regard sur le monde, un nouveau cadrage du réel visuel.

On connaît la bataille doctrinale qui va opposer Le Corbusier à celui qu'il avait dans un premier temps pris pour maître, Auguste Perret. Perret a, sans discontinuer, conçu ses ouvertures comme des fenêtres en hauteur, verticales, comme une projection vers l'extérieur de l'homme debout, ceci conformément à la tradition classique et aux anciennes techniques de construction, économes en linteaux et attentives à contenir les descentes de charge. Le Corbusier défend quant à lui la «fenêtre en longueur $»^{10}$. Cette position lui était offerte par le choix structurel ${ }^{11}$, mais lui était dictée par une volonté culturelle $^{12}$. En effet, la fenêtre en longueur introduit, dans la relation des habitants avec l'extérieur, plus de lumière, de ciel, de paysage, un rapport plus large avec le « dehors ", l'environnement, la nature. Ce caractère novateur pouvait à lui seul justifier la défense de l'ouverture horizontale. Plus profondément, le choix effectué par les architectes du Mouvement moderne reposait sur l'idée d'une relation nouvelle avec l'extérieur. La fenêtre ne devait plus être seulement un moyen d'aération et d'éclairage ou de guet furtif, mais devenait un moyen d'intégrer l'extérieur dans l'intérieur du logement en faisant du dehors un spectacle légitime dans lequel l'habitant était l'acteur du choix des points de vue offerts par une vaste scénographie. La fente horizontale correspondait par ailleurs, d'après les architectes, à la vision humaine, plus large que haute. On la retrouve dans les visières des casques d'armures. Paul Virilio établit, par la remarquable série de ses photographies (fig. $\mathbf{n}^{\circ} \mathbf{3}$ ), une relation qu'il qualifie d'« anthropomorphique » entre les meurtrières des blockhaus et des tours d'observation avec la tête humaine (p. 90, 91, 92 et 93, le cadrage isole deux meurtrières qui deviennent deux yeux) ou le casque (tours et postes de guets, par exemple p. 95, 97...). La Tour de direction de tir, qu'il photographie sous plusieurs angles, est très démonstrative de la modernité de ces fentes horizontales. L'alternance des bandeaux et des vides, y compris dans leurs proportions, est comme une prémonition du Guggenheim Museum de Frank Lloyd Wright à New York. En réalité, il n'y a probablement pas de relation directe entre le Mur et telle ou telle œuvre ou théorie du mouvement moderne. Mais il serait étonnant qu'il ne s'agisse que de simples coïncidences. La fenêtre en longueur était alors une réalité très répandue, en particulier en Allemagne. Sa performance fonctionnelle (balayage du regard dans l'immeuble d'habitation, du tir dans la tourelle) l'impose dans le civil comme dans le militaire et même lui confère un statut d'icône de la modernité. 


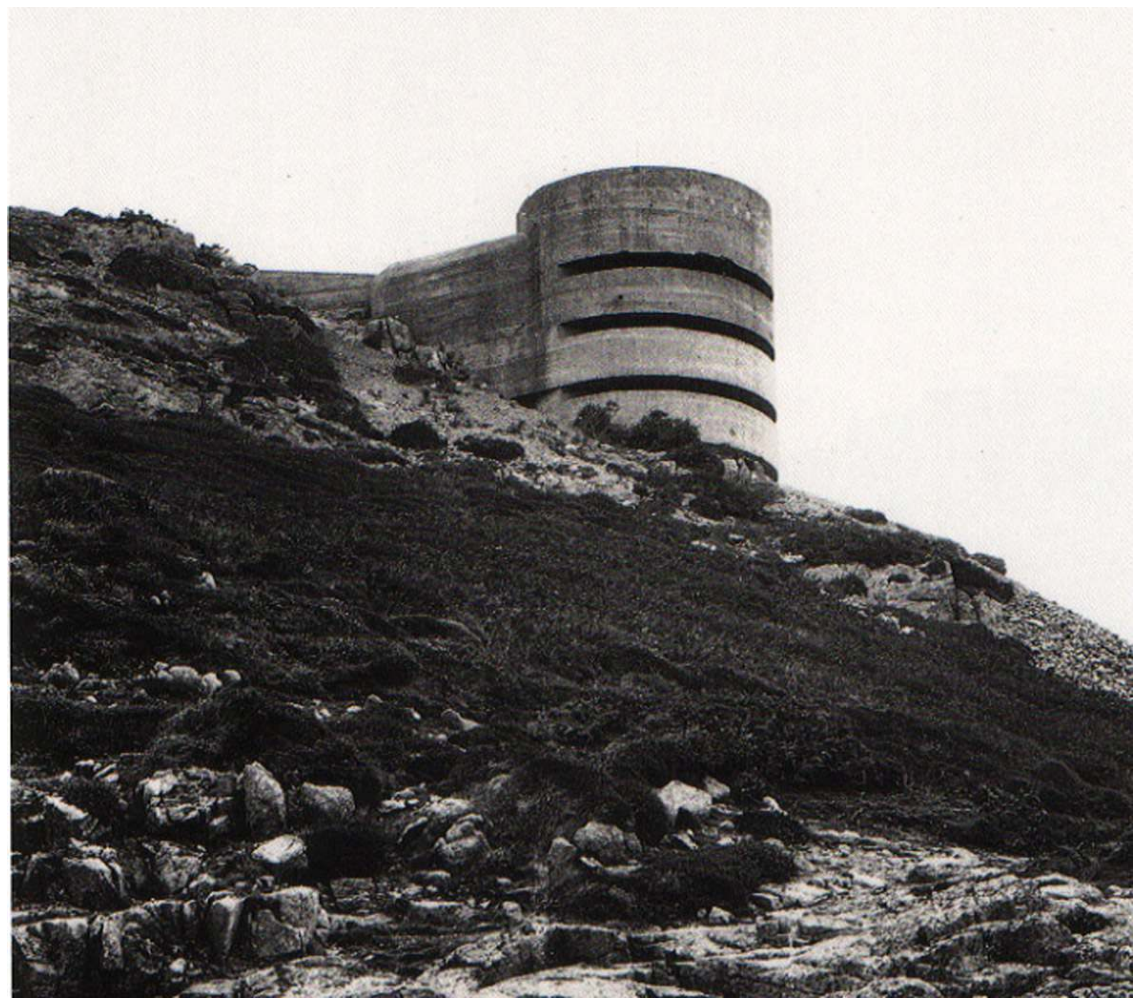

Tour d'observation. VIRILIO, Paul. BUNKER ARCHÉoLogIE, p. 113. D.R.

Autre comparaison, tout aussi hypothétique en tant que causalité, mais vraisemblable comme convergence: celle que l'on peut établir avec l'écran du cinématographe. Les formats de pellicule et de projection ont été nombreux et changeants durant toute l'histoire du cinématographe. L'image large fait partie des formats disponibles depuis les origines du cinéma. Marey utilise pour son chronophotographe le format 1 x 2,66. Toutefois, dès 1909 , le $35 \mathrm{~mm}$, avec des fenêtres plus ou moins carrées, s'impose comme le standard le plus couru. En 1927, Henri-Jacques Chrétien invente un dispositif anamorphoseur qui permet d'obtenir une image de 1 x 2,33. Claude Autant-Lara l'expérimentera en 1928 dans son court-métrage Construire un feu. Ce procédé annonce le Cinémascope, sous brevet de la Twentieth Century Fox, qui compte, grâce à lui, résister à la concurrence croissante de la télévision. Le premier film « en cinémascope » sera La Tunique (The Robe) d'Henri Koster, projeté en 1953.

14 Le succès du cinémascope est immédiat et tous les studios vont s'employer à proposer sous diverses appellations (Vistavision, Panavision, Cinérama...) une vision large, spectaculaire, favorable aux grands espaces, au concours de foules, au lyrisme qui contraste avec la petite lucarne carrée de la télévision. Par la suite, l'image cathodique puis numérique va suivre le même chemin et aujourd'hui c'est le format 16/9 et le grand écran mural qui apparaissent comme les standards universels.

On dispose de peu d'images prises depuis l'intérieur des meurtrières horizontales, sauf justement dans les films qui doivent restituer les deux "points de vue »: celui des attaquants qui affrontent un monolithe et celui des défenseurs qui perçoivent un panorama. Les ouvertures dans le béton contribuent donc à inscrire les édifices du Mur dans ce nouveau rapport à l'extérieur, la vision horizontale, synonyme de modernité. 
16 Le Mouvement moderne et sa "fenêtre en longueur", l'écran panoramique du cinémascope, les films sur le débarquement sont autant de «correspondances » qui permettent de percevoir le Mur comme une production de la modernité.

\section{Le Béton}

17 Matériau emblématique de la modernité, davantage que l'acier, le béton est vanté et utilisé par les architectes du Mouvement Moderne (et d'autres bien entendu) pour sa plasticité, comme une " pierre ", souple un premier temps, résistante ensuite. Dans le cas des fortifications allemandes, c'est sa simplicité de mise en œuvre et son coût qui le feront préférer à la pierre. Coulé en couches épaisses, le béton du Mur n'offre pas les voiles et porte-à-faux des édifices civils. Il devient la nouvelle image de la solidité alors que jusque-là la symbolique militaire de la résistance aux coups étaient incarnée par le cuir (les cuirasses), le métal (les casques, les armures, les blindages) et la pierre (les forts).

S'ajoute un autre lien de parenté avec la modernité, dans le réel comme dans l'imaginaire, celui de sa fragilité, qualité qui peut paraitre paradoxale alors qu'on vient d'évoquer sa «dureté ». Oxydation du fer, carbonatation de l'agrégat, lèpre et champignons divers attaquent le Mur de l'Atlantique comme ils attaquent le Capitole de Chandigarh ${ }^{13}$ et d'autres réalisations de la modernité.

La souffrance du béton est l'une des préoccupations principales des acteurs de la reconversion du Mur. Comment rendre accessible au public des lieux qui sont menacés non pas d'effondrement (ce qui dans bien des cas résoudrait le problème par le vide) mais de délitement ? Cette problématique est particulièrement présente dans les opérations de reconversion des bases de sous-marins où les hauteurs des volumes (les bases font jusqu'à $24 \mathrm{~m}$ de hauteur), spécialement celles des alvéoles, rendent très dangereuse la moindre chute de matériau.

\section{La monumentalité}

20 Les ouvrages les plus spécifiques du Mur relèvent du camouflage, de l'enfouissement, et de ce fait, sont peu ou mal visibles. Ils ne possèdent donc pas à priori les attributs habituels de la monumentalité : localisation emblématique, visibilité, lien affiché avec des valeurs sociales éminentes. Certains ouvrages, toutefois, par leurs dimensions et/ou leur architecture se donnent à lire comme des édifices singuliers, « veufs et orphelins » au sein de la famille des immeubles ordinaires, expressifs et impressionnants; en particulier les tours et les bases de sous-marins. Si fonctionnellement les édifices du Mur ne cherchent pas la monumentalité, formellement leur masse et leur originalité les posent dans l'espace urbain comme des signes peut-être énigmatiques, certainement imposants.

21 Paul Virilio n'hésite pas à parler de "monuments militaires » et même liturgiques, de casemates ressenties comme des "petits temples sans religion"; il relève ainsi «le caractère quasi - religieux des autels de plage, abandonnés au jeu des enfants $\aleph^{14}$. Il note par ailleurs que Speer, obsédé par la volonté de laisser des traces durables du Reich, de témoigner de sa grandeur par des ruines qui resteraient admirables dans les siècles à venir, assigne aux édifices du Mur «le rôle imparti aux monuments de prestige, témoignant moins de la puissance du III Reich que de sa hantise de la disparition ${ }^{15}$. 
Virilio esquisse à peine la question des référents de cette monumentalité, mais se souvient des échos qu'avait suscités sa première découverte du Mur : «Toute une série de réminiscences culturelles me saisirent: les mastabas, les tombes étrusques, les structures aztèques... $»^{16}$. Roz corrobore lui aussi l'écho aztèque.

3 Nous avons évoqué plus haut l'analogie avec le modernisme lyrique du Wright de la dernière période. Nous devons également établir un rapprochement (de fait car Le Corbusier, sensible aux silos américains, ne fait guère référence aux édifices militaires dans son œuvre écrite) entre l'évidente monumentalité des façades des bases de sousmarins et la scansion des pilotis du rez-de-chaussée des Unités d'habitation, en particulier celle de Marseille.

Quoi qu'il en soit de la légitimité à l'origine de la monumentalité des édifices du Mur, leur fonction actuelle les instaure en monuments. C'est-à-dire, au sens que lui donne l'Unesco, comme œuvre portant témoignage d'un "moment» de la culture humaine et en cela constitutifs de l'héritage de l'humanité. Mais aussi en monument au sens "romain», urbanistique du terme, d'édifice singulier venant scander le parcours urbain, à l'instar des arcs de triomphe, des colonnes commémoratives, des portes de villes, etc.

\section{Un paysage spécifique}

Alain Demangeon et Bruno Fortier, dans un ouvrage qui fit grande impression dans l'univers culturel de l'architecture ${ }^{17}$, ont décrit le processus conceptuel qui a fait des réalisations militaires (en l'occurrence les arsenaux) les ferments d'une transformation radicale des modes de pensée qui aboutira à l'architecture du Mouvement moderne deux siècles plus tard.

« ... la spatialité des arsenaux... il ne s'agit en l'occurrence ni d'une forme ni d'un modèle ni même d'un vocabulaire ou d'un style que l'on retrouverait dans tous les projets d'arsenaux, mais d'une manière de répartir les vides et de marquer les pleins, de dilater l'espace... et de le découper partout où il peut être utile, d'effacer les typologies sous des régularités plus diffuses et de dématérialiser les constructions pour en faire de simples écrans. D'imaginer en somme, un parfait négatif de ce qu'avaient été les villes classiques » ${ }^{18}$.

Le Mouvement moderne a tenté avec un succès mitigé et contesté d'inventer une nouvelle relation entre les bâtiments et l'espace urbain qui les environne en s'émancipant de l'alignement sur rue, en dégageant les alentours immédiats de l'édifice (voire en supprimant le rez-de-chaussée) au profit d'espaces verts, en établissant une nouvelle typologie des voies de circulation...

Comme toute fortification, les constructions du Mur de l'Atlantique sont entourées d'un espace libre plus ou moins étendu, glacis, dégagement des perspectives, distances de sécurité... Cet isolement est renforcé le plus souvent par l'implantation dans des zones non construites (ou déconstruites), face à la mer. Rien dans le schéma territorial du Mur qui rappelle la « ville traditionnelle » et son décor, sa densité, sa logique viaire. L'espace distendu, discontinu, clairsemé de Mur n'est pas sans rappeler les vastes esplanades de la ville moderne, portées à leur paroxysme à Brasilia. C'est là un espace particulier que Virilio appelle "le paysage de la guerre ${ }^{19}$. Ultérieurement, ce paysage sera supplanté dans la mythologie collective par celui des cimetières militaires aux croix blanches strictement alignées sur le flanc des falaises, comme des parkings de supermarchés. 

rapporté aux propositions radicales des Modernes. Les exemples théoriques les plus connus sont la ville linéaire de Arturo Soria y Mata $(1886)^{20}$ et les plans de ville immeuble de Le Corbusier pour Alger et surtout Rio de Janeiro (fin des années 1920) ${ }^{21}$. Le Mur aurait-il réalisé le rêve de la ville linéaire que ces grands visionnaires n'avaient pas pu mettre en œuvre?

30 Toutefois, ces caractéristiques du Mur qui montrent combien les fortifications sont en écho avec la modernité des années 1930 et 1940, n'effacent pas des différences importantes. Les fortifications ne respectent pas (faut-il s'en étonner ?) les « cinq points » de l'architecture moderne. Le pilotis est exclu de l'architecture fortifiée. Alors que, par ce dispositif, les édifices modernes tentent de s'émanciper de la pesanteur et de l'enracinement ${ }^{22}$, les fortifications au contraire entretiennent avec la terre, le sol, la roche une relation intime; elles s'y enfouissent, s'en recouvrent, s'y camouflent. Autre différence fondamentale : l'ordonnancement de l'enveloppe des fortifications n'est pas le reflet de l'organisation intérieure, il ne l'exprime ni ne l'instrumente. Au contraire la morphologie émergée constitue un bouclier qui protège la cavité et un masque qui cache l'ampleur et la logique des locaux. Rien de la complexité interne ne transparaît. L'enveloppe des édifices du Mur, si elle est en contraste avec les préceptes du Mouvement Moderne, se rapproche en revanche de la modernité du XXI e siècle, qui habille les tours de coques et de treillis quasiment autonomes et qui les camoufle même sous une végétation pariétale.

\section{Un patrimoine démilitarisé entre éthique et authenticité}

31 Aux qualités morphologiques que nous venons d'évoquer s'ajoute dans le processus de patrimonialisation le « contenu » des édifices : la guerre, les dispositifs et les machines de destruction, l'occupation allemande, le débarquement, les Alliés, la victoire... Malgré l'évidente destination des édifices du Mur de l'Atlantique, on constate que leurs fonctions sont rarement prises en compte dans leur remise à jour : à Saint-Nazaire c'est l'univers des paquebots qui est évoqué dans le musée de l'Escale Atlantique (fig. $\left.\mathbf{n}^{\circ} \mathbf{4}\right)$; à Lorient, si les bases sont ouvertes à la visite, les programmes tournent davantage autour de la voile et de la mer que de la guerre; à Bordeaux ce sont des expositions temporaires, de l'événementiel et des répétitions théâtrales et chorégraphiques qui occupent le quart des surfaces accessibles au public, etc. Dans tous les cas de figure, la reconquête des bases abandonnées vise avant tout la recomposition urbaine et l'accroissement de l'offre de loisirs en profitant d'une opportunité d'appropriation de friches de plus en plus rares, pour répondre à la fascination croissante du public pour les constructions atypiques, le goût de la ville mêlée à l'eau (les références vénitiennes ne sont pas absentes dans le traitement des alvéoles ); seule la dimension militaire est comme absente, oubliée ou estompée, des programmes de valorisation des sites allemands fortifiés. 
Figure 4

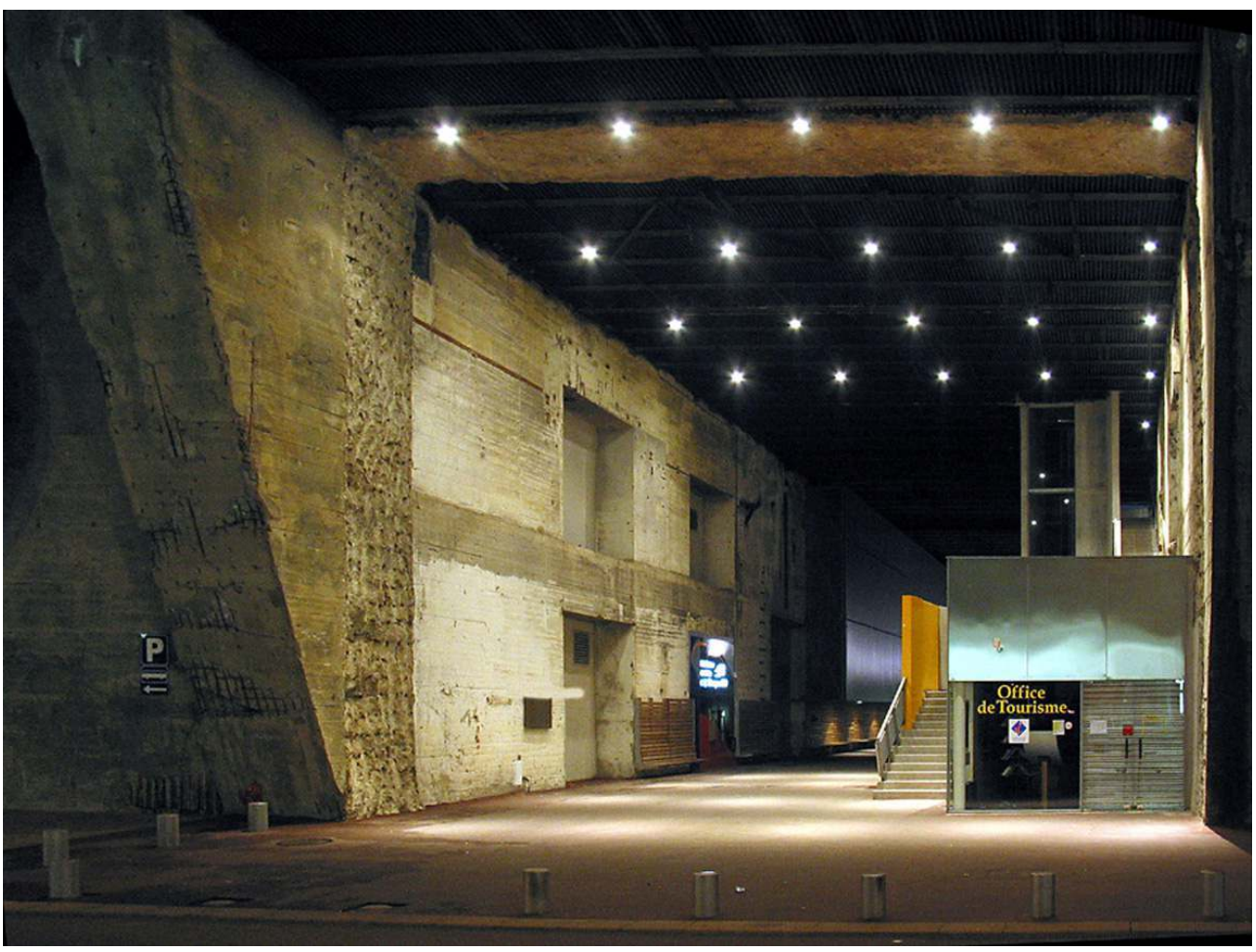

Saint-Nazaire, entrée de « L'escale atlantique ». Phot. mairie de Saint-Nazaire. délectation clandestine; les "musées des horreurs ", les films du même genre, les jeux informatiques en sont des manifestations. L'Unesco a inscrit au patrimoine mondial nombre de témoignages de la folie humaine, indépendamment de leur intérêt architectural ou urbanistique, le plus souvent faible ou inexistant. L'un des premiers exemples date de 1978: c'est celui de l'île de Gorée, au large de Dakar, retenu comme "symbole de l'exploitation humaine » $^{23}$ et transformée en musée. En 1979 le camp de concentration nazi de Auschwitz-Birkenau en Pologne, a été inscrit sur la liste en tant que «symbole de la cruauté de l'homme pour l'homme au XXe siècle ». En 1996, c'est le bâtiment en partie rescapé du crime atomique d'Hiroshima qui est sanctuarisé par l'Unesco, comme «symbole dur et puissant de la force la plus destructrice que l'homme ait jamais créée ». En 2010 enfin, l'Unesco a retenu les « sites de bagnes australiens », soit 11 colonies pénitentiaires où étaient déportés condamnés britanniques et orphelins, bagnes qualifiés de «meilleurs exemples survivants de la déportation de criminels à grande échelle ».

34 Les initiatives commémoratrices hors Unesco sont tout aussi nombreuses. Ainsi, à Kobé, un mémorial rappelle le tremblement de terre en conservant, comme trace mémorielle, une partie des quais ruinés. Aujourd'hui New York cherche les formes à donner au site à l'attentat du 11 septembre 2001. 

les détruire, sont devenus, du fait de leur inaltérabilité, des témoins très ou trop visibles. Très visibles du fait de leur monumentalité et de leur étrangeté, trop visibles car leur persistance les rend comme éternels et de leur fonction incontournable.

\section{La base de Saint-Nazaire}

La base de Saint-Nazaire, une des plus importantes avec celle de Lorient, comporte 16 alvéoles, occupe 3,7 hectares et s'étire sur $295 \mathrm{~m}$ le long du port ; elle est complétée par une écluse fortifiée. La dalle de toiture d'une épaisseur de $4 \mathrm{~m}$ a résisté à tous les bombardements (fig. $\mathbf{n}^{\circ} \mathbf{5}$ ).

Figure 5

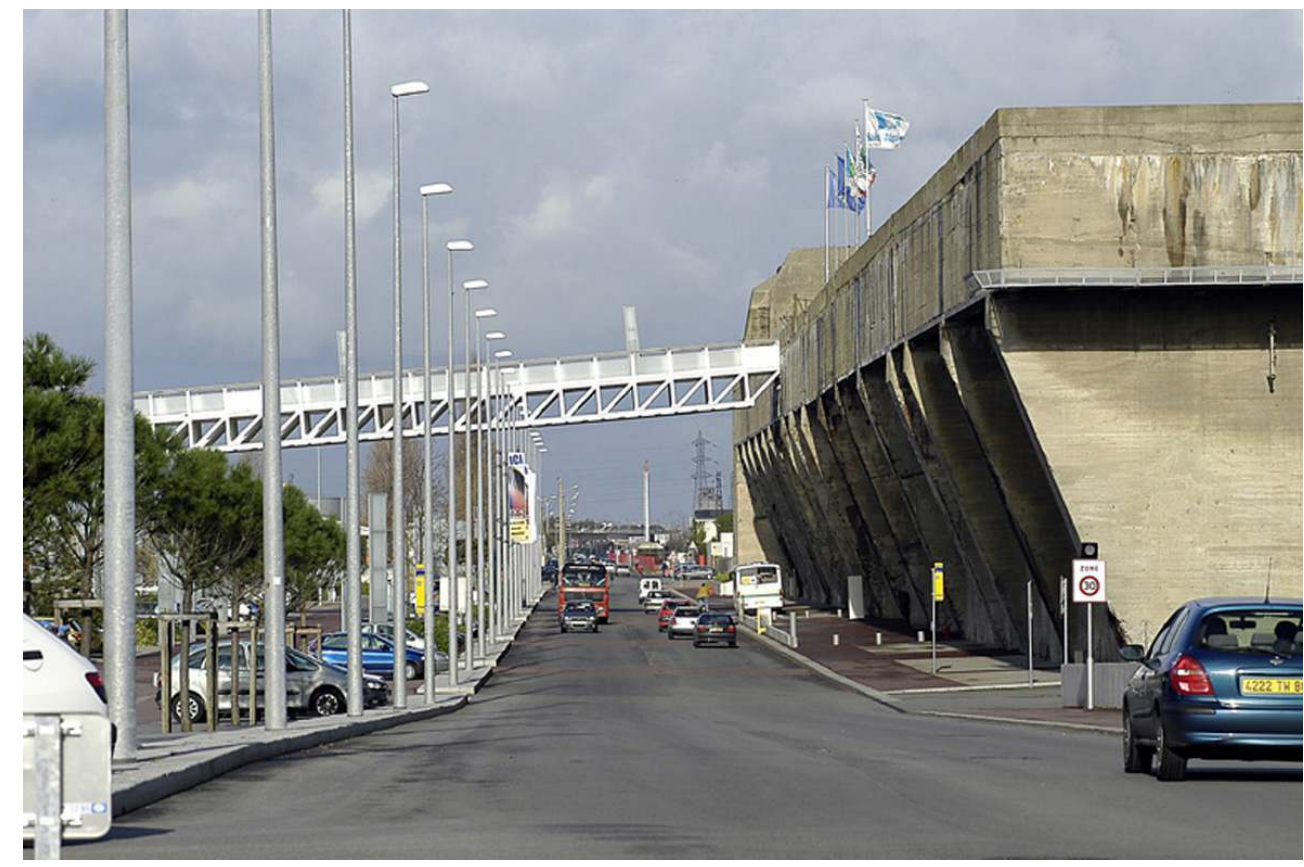

Base de sous-marins de Saint-Nazaire. Façade côté ville et passerelle de Solà Moralès. Phot. Claude Prelorenzo.

Longtemps sans reconnaissance collective, dévolue à la culture du soja dont la pestilence accroissait son rejet, elle devint la pièce essentielle d'une recomposition urbaine lorsque le maire, audacieux, décida dès 1989 de révolutionner la structure urbaine de la reconstruction conçue par Le Maresquier qui tournait le dos au port pour la tirer vers les bassins et la mer. Après une première "résurrection » obtenue grâce au succès de la « Nuit des Docks » (1991) conçue par l'artiste en lumière Yann Kersalé, la Ville lance, en 1994, une consultation pour faire de Saint-Nazaire une "ville-port" attractive. C'est l'architecte espagnol Manuel de Solà Moralés i Rubio qui l'emporte et qui réalisera les premières et décisives opérations: rénovation des alvéoles centrales transformées en Musée du paquebot, "L'escale Atlantique ", et création d'une gigantesque rampe qui conduit le public directement sur le toit devenu ainsi belvédère. En 2005, le projet « Villeport 2 » est confié à l'agence berlinoise Finn Geipel \& Giulia Andi. Le programme accentue 
les fonctions de loisirs, conduisant les architectes à ouvrir les alvéoles 13 et 14 sur la ville et à y installer des équipements comme le LiFE (Lieu International des Formes Émergentes) et le VIP, destinés à accueillir spectacles, concerts et événements. La réussite architecturale, le succès public et l'effet de recomposition urbaine sont remarquables. Mais on peut considérer que là comme ailleurs ils sont obtenus par un détournement de la fonction mémorielle. Danser et promener ses enfants sur des lieux destinés à tuer ne va pas de soi. Retenons cependant que cette dimension de souvenir négatif n'est pas complètement absente puisque la mutation citadine s'accompagne d'actions, concrétisant la réconciliation avec l'Allemagne unifiée : sur le toit est en effet installée en 2007 une structure appelée «le radôme» qui contient un radar de l'aérodrome de Tempelhof (Berlin), offert par le ministère allemand de la Défense.

\section{La base de Lorient}

Véritable complexe fortifié, ce que l'on appelle par commodité de langage la «base de sous-marins » de Lorient-Keroman ${ }^{24}$ est composée de trois bases totalisant un linéaire de plus de $370 \mathrm{~m}$, sur $25 \mathrm{~m}$ de hauteur, de deux dômes cathédrales (Dom Bunker), d'une quatrième base inachevée et d'importants casernements... (fig. $\mathbf{n}^{\circ} \mathbf{6}$ )

Figure 6

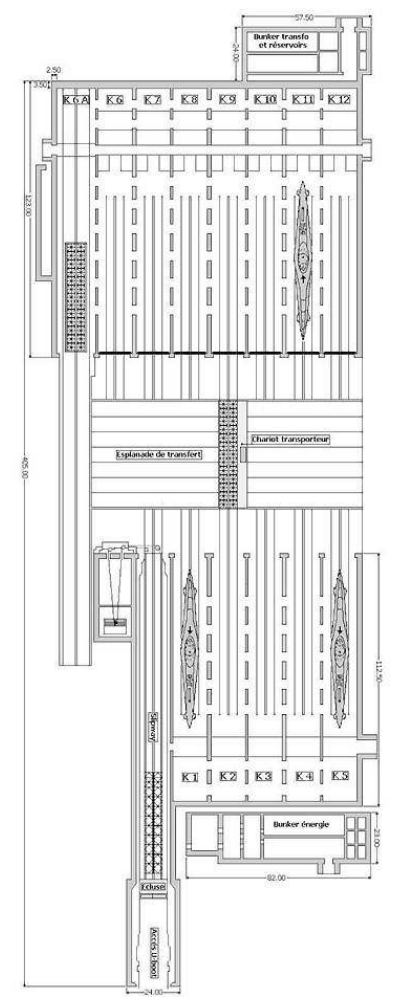

Base de sous-marins de Lorient, plan des abris « $\mathrm{K} \mid$ » et « $\mathrm{K} \|$ ». Dessin Patrick Fleuridas, D.R.

L'ensemble de cette « forteresse » allemande a été conservé, malgré son ampleur (et donc son coût d'entretien) et le déficit assumé de foncier que cela représentait. Comme les autres bases, celle de Lorient est entièrement gérée par la Ville qui ne semble pas souhaiter une reconnaissance patrimoniale officielle. Dès les années 1990, les concours mettent l'accent sur ce qui va être la particularité de l'action urbanistique sur ce 
patrimoine : installer au milieu des activités culturelles, des entreprises contemporaines, réaliser un quartier actif. Ce parti pris, pas toujours bien compris en son temps, en particulier par les services de l'État, montre aujourd'hui sa fertilité. Entre les blocs ont surgi de nouvelles architectures: les grands hangars blancs de Jean-François Revert, la Tour et la Cité de la Voile Éric Tabarly de Jacques Ferrier et en retrait des quais, bientôt reliés au re-développement du secteur du port de pêche, les bâtiments d'entreprises privées en relation avec la mer et l'innovation.

41 Il existe à l'intérieur de l'un des bunkers un " Musée sous-marin », mais contrairement à ce à quoi l'on pourrait s'attendre, ce musée, fort intéressant et original au demeurant, ne porte pas sur les sous-marins, à l'exception notable de la présentation d'un simulateur de plongée de 1942, mais donne à connaître et comprendre la plongée et le monde des profondeurs marines.

Le seul sous-marin présenté sur le site est anachronique et trompeur puisqu'il s'agit du Flore, sous-marin français mis en service en 1964.

Il serait présomptueux de vouloir conclure à propos d'un phénomène aussi particulier que le Mur de l'Atlantique et ses édifices car le processus sinon de patrimonialisation, du moins d'intégration, est en cours et connaîtra certainement des développements et des réorientations. La situation est en effet paradoxale et peu fréquente: engager un processus d'appropriation d'un patrimoine doublement hétérogène et émettant un message peu propice aux loisirs et au tourisme; en effet, d'une part il est l'œuvre d'étrangers et à l'époque ennemis, et d'autre part il est la mémoire de la défaite militaire et de la destruction de la ville par les troupes amies! Les différents programmes déjà réalisés ou qui viendront, doivent donc nécessairement adoucir, par la mise en service d'activités appréciées des citoyens, la réalité de ces monuments dont on ne peut mettre en valeur la fonction commémorative, tout au moins pour un certain temps encore.

Au travers des deux exemples de Saint-Nazaire et de Lorient, apparaît clairement le mode spécifique de patrimonialisation des bases et plus largement des édifices du Mur de l'Atlantique. D'une part les bâtiments se sont assez bien conservés par eux-mêmes, leurs dimensions autorisant l'interdiction d'accès dans les zones dangereuses, d'autre part leur étrangeté a joué comme un stimulant dès lors que le souvenir des bombardements s'est éloigné ; enfin leur situation aux marges de la cité a permis de développer un centre urbain secondaire attractif. Reste que la dimension guerrière, violente et meurtrière des dispositifs, très présente au cinéma ${ }^{25}$ est partout estompée au profit des aspects pharaoniques des construction, la beauté paysagère des sites d'enfouissement et la plastique séduisante des rondeurs de béton.

\section{NOTES}

1. - Cet article synthétise des recherches effectuées depuis 2000 dont il reprend en partie deux publications : PRELORENZO, Claude. The consolidation of Military Works in the Port Cities of France, in Aquapolis «Revitalisation of the Military Heritage of Maritime Cities ", $n^{\circ} 1 / 2000$, mars 2000. PRELORENZO, Claude. Le Mur de l'Atlantique dans la modernité, in Gennaro 
Postiglione (dir.) The Atlantic Wall, linear museum, EU Programme Culture 2000, multigrafié, octobre 2005.

2. - Les bases établies en France sont au nombre de cinq: Brest (1941-42), Lorient (Keroman, 1941-1944), Saint-Nazaire (1941-43), La Rochelle (La Palice, 1941-1943) et Bordeaux (Bacalan, 1941-1943); il convient de mentionner également celle, moins connue, de Marseille qui, inachevée, ne fut jamais en service.

3. - On consultera le site Internet créé à l'occasion de la recherche référencée supra: http:// www.atlanticwall.polimi.it/museum/

4. - Voir ROZ, Michel. Les bases de sous-marins en France. 1943-1993. Construction et reconversion, rapport de recherche, Plan Construction et Architecture, Ministère de l'Équipement, de l'Urbanisme et du Logement. Paris : s.d., circa. 1994.

5. - SANTANGELO, Andrea. Le mur de l'Atlantique en représentation, Gennaro Postiglione (dir.) The Atlantic Wall, linear museum, EU Programme Culture 2000, multigrafié, octobre 2005.

6. - SANTANGELO, Andrea. Le mur de l'Atlantique en représentation, Gennaro Postiglione (dir.) The Atlantic Wall, linear museum, EU Programme Culture 2000, multigrafié, octobre 2005.

7. - Michel Roz dans son rapport d'étude évoque, après Virilio, la référence aztèque à propos de la monumentalité des bases de sous marins.

8. - Comment écrire sur ce thème après Paul Virilio ? Il a non seulement initié les recherches sur le Mur, mais les a pratiquement achevées, parachevées même. Son style ne peut être égalé, ses photographies dépassées, son art d'associer science et poésie, objectivité et implication, repris. Le lecteur devra donc sans cesse et avant tout se reporter à son ouvrage : VIRILIO, Paul. Bunker archéologie. Paris : éditions du Demi-Cercle, 1991 (1 édition 1975).

9. - Le très officiel Vocabulaire de l'architecture, publié par le Ministère de la Culture français (en 1972), s'il consacre quelques pages illustrées à l'architecture militaire où abondent donjons et remparts à meurtrières verticales, ne montre qu'un seul dessin de meurtrière à embrasure horizontale, celle ménagée dans une canonnière. Voir Principes d'analyse scientifique. Architecture. Méthode et vocabulaire. Ministère des Affaires Culturelles. Inventaire Général des monuments et des richesses artistiques de la France. Paris: Imprimerie Nationale, 1992, 2 tomes.

10. - LE CORBUSIER. Les cinq points d'une architecture nouvelle, in Oeuvre complète, volume 1 1910-1929. Girsberger, 1964 ( $1^{\mathrm{e}}$ édition 1929), p. 128.

11. - «La construction à ossature permet... le percement des baies sans tenir compte des descentes de charge ». MONNIER, Gérard. Le Corbusier. Besançon : éditions de La manufacture, 1992, p. 32.

12. - On peut en trouver la preuve par le contraire dans le choix d'ouvertures carrées ou en fentes verticales qu'il effectue pour son propre « cabanon » de Cap-Martin.

13. - On se réfèrera, parmi les nombreuses publications, au séminaire qui s'est tenu à Chandigarh en 2002 : JOSHI, Kiran (editor). Corbusier's Concrete. Challenges of conserving moderne architecture. New Delhi : édition Chandigarh Perspectives, 2005.

14. - VIRILIO, Paul. Bunker archéologie. Paris : éditions du Demi-cercle, 1991 (1e édition 1975), p. 14.

15. - VIRILIO, Paul. Bunker archéologie. Paris : éditions du Demi-cercle, 1991 (1e édition 1975), p. 57.

16. - VIRILIO, Paul. Bunker archéologie. Paris : éditions du Demi-cercle, 1991 (1édition 1975), p. 11.

17. - DEMANGEON, Alain et FORTIER, Bruno. Les vaisseaux et les villes. Bruxelles : éditions Pierre Mardaga (collection Architecture + Archives), 1978.

18. - Ibid., p. 30.

19. - Ibid., p. 75. 
20. - SORIA Y MATA, Arturo. La Cité linéaire. Nouvelle architecture des villes. $1^{\mathrm{e}}$ édition Madrid 1886, fac-similé par l'École Nationale Supérieure des Beaux-Arts, Paris, 1984.

21. - LE CORBUSIER. Oeuvre complète, volume 2 1929-1934. Grisberger, 1964.

22. - N'oublions pas la fascination des Modernes pour le zeppelin, habitat volant, presque aussi forte que celle pour le paquebot, immeuble naviguant.

23. - Voir les commentaires de la liste du patrimoine mondial sur le site : http://whc.unesco.org/ fr/list.

24. - Voir le plan de la base de Lorient-Keroman : BRESSOL, Thierry. Souvenirs de mer, éd Internet : http://souvenirs-de-mer.blogdns.net/.

25. - On pense en particulier au célèbre film oscarisé Le jour le plus long, tourné en 1962 sur le thème du débarquement de Normandie (scénariste et auteur du roman : Cornelius Ryan).

\section{RÉSUMÉS}

Le Mur de l'Atlantique court de la frontière espagnole jusqu'au nord de la Norvège. Au sein de ce dispositif sont installées cinq bases de sous-marins: Dunkerque, Saint-Nazaire, Lorient, La Rochelle et Bordeaux. Après une période de purgatoire, liée aux souvenirs trop frais des bombardements qu'ils ont attirés sur les villes, les édifices du mur sont entrés dans la sphère de la culture et du tourisme. Nous posons alors la question suivante : de quoi ces édifices portent-ils témoignage, et nous envisageons plusieurs aspects : leur évident modernisme qui les situe à un moment de l'histoire de l'architecture, leur hétérogénéité qui fait de ces bâtiments des sortes d'ovni, sans aucun rapport ni d'échelle ni de forme avec l'architecture civile, une technique d'enfouissement et de camouflage génératrice d'un nouveau paysage. Mais ces édifices peuventils être exonérés de leur but guerrier? Quel est le message de leur recyclage dans le culturel, le festif ou le commercial ? Deux bases de sous-marins sont plus particulièrement présentées, celle de Saint-Nazaire et celle de Lorient.

The Atlantic Wall stretches from the Spanish border to northern Norway. Within this system there are five bases for submarines: Dunkirk, Saint-Nazaire, Lorient, La Rochelle and Bordeaux. After a period of purgatory, with the painful memories of the wartime air raids which destroyed the cities around the bases, the buildings which the Atlantic Wall comprises have now entered the spheres of culture and tourism. What do these buildings bear witness to? Their frank modernism puts them at a precise moment in the history of architecture. Their heterogeneity makes them like UFOs, without any relation of scale or form to civil architecture. Their specific techniques of dissimulation and camouflage created new landscapes. But can these buildings be freed from the memories of their original military purpose? What message is delivered when they are recycled for new, cultural, leisure or commercial usages? Two submarine bases at SaintNazaire and at Lorient, are focussed on in this article. 
INDEX

Mots-clés : bases de sous-marins, Bordeaux, Brest, fortifications, La Rochelle, Lorient, modernité, Mur de l'Atlantique, Saint-Nazaire, XXe siècle

Keywords : Atlantic Wall, fortification, Heritage: Submarine Bases and the Atlantic Wall, modernity, submarine base, Twentieth century

\section{AUTEUR}

\section{CLAUDE PRELORENZO}

Maître de conférences à l'École des Ponts - Paris Tech Chercheur associé au LIAT (ENSA Paris Malaquais) Secrétaire général de la Fondation le Corbusier Claude.prelorenzo@free.fr 